NASA Technical Memorandum 4651

\title{
Numerical Modeling of a Cryogenic Fluid Within a Fuel Tank
}

Donald S. Greer

Dryden Flight Research Center

Edwards, California

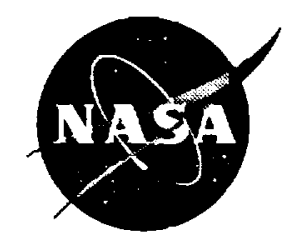

National Aeronautics and Space Administration Office of Management

Scientific and Technical Information Program 1994 



\title{
NUMERICAL MODELING OF A CRYOGENIC FLUID WITHIN A FUEL TANK
}

\author{
D.S. Greer" \\ NASA Dryden Flight Research Center \\ P.O. Box 273 \\ Edwards, California 93523-0273
}

\begin{abstract}
The computational method developed to study the cryogenic fluid characteristics inside a fuel tank in a hypersonic aircraft is presented. The model simulates a rapid draining of the tank by modeling the ullage vapor and the cryogenic liquid with a moving interface. A mathematical transformation was developed and applied to the NavierStokes equations to account for the moving interface. The formulation of the numerical method is a transient hybrid explicit-implicit technique where the pressure term in the momentum equations is approximated to first order in time by combining the continuity equation with an ideal equation of state.
\end{abstract}

\section{Nomenclature}

$\begin{array}{ll}a & \text { Van der Waals constant, } \mathrm{Pa} \mathrm{m}^{6} \\ b & \text { Van der Waals constant, } \mathrm{m}^{3} \\ c & \text { speed of sound, } \mathrm{m} / \mathrm{sec} \\ \text { FI } & \text { flux, radial direction } \\ \text { FJ } & \text { flux, circumferential direction } \\ g & \text { gravity, } \mathrm{m} / \mathrm{sec}^{2} \\ h & \text { enthalpy, J/kg } \\ k & \text { conductivity, J/m sec K } \\ \text { MSF } & \text { Mach scaling factor, } c_{\text {real }} / c_{\text {numerical }} \\ \text { NIST } & \begin{array}{c}\text { National Institute of Standards and Technol- } \\ \text { ogy, Gaithersburg, Maryland }\end{array}\end{array}$

Research Engineer.

Copyright 1994 by the American Institute of Aeronautics and Astronautics, Inc. No copyright is asserted in the United States under Title 17, U.S. Code. The U.S. Government has a royalty-free license to exercise all rights under the copyright claimed herein for Governmental purposes. All other rights are reserved by the copyright owner.

\begin{tabular}{|c|c|}
\hline$P$ & pressure, $\mathrm{Pa}$ \\
\hline$q$ & heat flux, $\mathrm{J} / \mathrm{m}^{2} \mathrm{sec}$ \\
\hline $\mathbf{R}$ & radius relative to interface, $\mathrm{f}(\theta, t), \mathrm{m}$ \\
\hline $\mathbf{R}$ & fluid constant, J/kg K \\
\hline $\mathbf{R}^{*}$ & ideal fluid constant, $\mathrm{J} / \mathrm{kg} \mathrm{K}$ \\
\hline $\mathbf{r}$ & radial coordinate, $m$ \\
\hline$r$ & transformed radius coordinate, $r / R$ \\
\hline$T$ & temperature, $\mathbf{K}$ \\
\hline$t$ & time, sec \\
\hline$V$ & velocity, $\mathrm{m} / \mathrm{sec}$ \\
\hline$\Delta r$ & distance between radial grid points, m \\
\hline$\Delta \vartheta$ & $\begin{array}{l}\text { distance between circumferential grid points, } \\
\text { rad }\end{array}$ \\
\hline$\alpha$ & thermal diffusivity, $\mathrm{m}^{2} / \mathrm{sec}$ \\
\hline$\mu$ & viscosity, $\mathrm{kg} / \mathrm{m} \mathrm{sec}$ \\
\hline $\mathbf{v}$ & specific volume, $\mathrm{m}^{3} / \mathrm{kg}$ \\
\hline$\theta$ & circumferential coordinate, rad \\
\hline$\rho$ & density, $\mathrm{kg} / \mathrm{m}^{3}$ \\
\hline$\vartheta$ & transformed circumferential coordinate, rad \\
\hline \multicolumn{2}{|c|}{ Superscripts } \\
\hline $\mathbf{n}$ & time step index \\
\hline \multicolumn{2}{|l|}{ Subscripts } \\
\hline $\mathrm{i}, \mathrm{j}$ & spacial coordinate indexes \\
\hline
\end{tabular}




$\begin{array}{ll}i+, i & \text { spacial coordinate at } i+\frac{1}{2}, i-\frac{1}{2} \\ j+, j & \text { spacial coordinate at } j+\frac{1}{2}, j-\frac{1}{2} \\ r, r & \text { radial direction } \\ s & \text { constant entropy } \\ \theta, \vartheta & \text { circumferential direction }\end{array}$

\section{Introduction}

Liquid hydrogen has long been considered one of the most advantageous fuels for hypersonic aircraft. The National Advisory Committee on Aeronautics (NACA), Lewis Flight Propulsion Laboratory, Cleveland, Ohio, began to study hydrogen as a fuel source in the 1950's. The NACA study included a flight test program with a U.S. Air Force B-57 which had one engine modified for operation with hydrogen fuel. ${ }^{I}$ The test program was successful, and the engine typically operated at an altitude of $50,000 \mathrm{ft}$ and Mach 0.75 for $17 \mathrm{~min}$ with hydrogen fuel. Since this initial test program, there have been several studies to investigate the aspects of liquid hydrogenfueled aircraft. An excellent review is presented by Brewer. $^{2}$

Developing reusable flight-weight cryogenic fuel tanks is one of the most difficult challenges in designing advanced hypersonic aircraft and the next generation of spacecraft. The most recent cryogenic fuel tank studies were conducted for the National Aero-Space Plane. McDonnell Douglas Corporation, St. Louis, Missouri, constructed and tested with liquid hydrogen a 3600-liter, graphite-reinforced, epoxy thermoset composite tank. ${ }^{3}$ The General Dynamics Space Systems Division, San Diego, California, also constructed and tested a 2600-liter graphite and epoxy liquid hydrogen tank. ${ }^{4}$ Presently, NASA Dryden Flight Research Center, Edwards, California, is involved in a research program to continue the study and to increase understanding of liquid hydrogen cryogenic fuel tanks. The initial phase of the program includes the design of a test facility and the development of a generic research cryogenic tank (fig. 1) for initial testing and verification of analytical models. Testing of the tank will simulate the severe aeroheating effects of hypersonic flight by subjecting the tank exterior to large heat fluxes. Details of the generic research cryogenic tank and test setup have been reported. ${ }^{5}$

These hypersonic cryogenic fuel tanks must be able to survive the thermal gradients of exterior gaseous aeroheating temperatures as high as $7000 \mathrm{~K}$ and internal cryogenic temperatures as low as $20 \mathrm{~K}$. See reference 6 for a detailed discussion on hypersonic aerodynamic heating. The understanding of the cryogenic fluid dynamic motion inside the tank is essential to predict and analyze the thermal stress gradients though the tank wall. The fluid dynamic model will predict cryogenic fluid temperatures, heat-transfer coefficients on the inner tank wall, and stratification effects in the ullage gas. An analysis using SINDA'85/FLUINT, which showed that these are the relevant interior parameters in determining the fuel tank thermal stresses, has been performed. ${ }^{5}$

The generic research cryogenic tank which this model was developed to simulate has met with budgetary constrains, and test data for verification of the model are unavailable. Comparison of the model with test data will be the subject of future efforts. This paper presents the computational fluid dynamic modeling development effort to simulate the generic research cryogenic tank when subjected to high extemal heat flux and rapid draining. The numerical methods were developed with emphasis on increasing the time step to reduce computer resources.

\section{Model Description}

Figure 2 shows the cross-sectional area of the generic research cryogenic tank modeled two dimensionally in polar coordinates. The interface between the cryogenic liquid and the ullage vapor is depicted as a dark horizontal line. This interface moves down as the tank is drained of liquid. The liquid exits the model through the bottom drain port, and ullage gas enters the model through the top pressurization port. The polar coordinate system was chosen to be relative to the moving interface to facilitate a coordinate transformation. The model employs the full set of Navier-Stokes equations with the exception that energy dissipation is neglected in the energy equation. For completeness, the applicable equations are given below.

Continuity

$$
\frac{\partial \rho}{\partial t}+\frac{\partial\left(\rho r V_{\mathrm{r}}\right)}{\partial \mathrm{r}}+\frac{1}{\mathrm{r}} \frac{\partial\left(\rho V_{\theta}\right)}{\partial \theta}=0
$$

Radial momentum

$$
\begin{aligned}
\rho( & \left.\frac{\partial V_{\mathrm{r}}}{\partial t}+V_{\mathrm{r}} \frac{\partial V_{\mathrm{r}}}{\partial \mathrm{r}}+\frac{V_{\theta}}{\mathrm{r}} \frac{\partial V_{\mathrm{r}}}{\partial \theta}-\frac{V_{\theta}^{2}}{\mathrm{r}}\right) \\
\quad & \frac{1}{\mathrm{r}} \frac{\partial}{\partial \mathrm{r}}\left(\mathrm{r} \mu\left[2 \frac{\partial V_{\mathrm{r}}}{\partial \mathrm{r}}-\frac{2}{3}\left[\frac{1}{\mathrm{r}} \frac{\partial\left(\mathrm{r} V_{\mathrm{r}}\right)}{\partial \mathrm{r}}+\frac{1}{\mathrm{r}} \frac{\partial V_{\theta}}{\partial \theta}\right]\right]\right) \\
& +\frac{1}{\mathrm{r}} \frac{\partial}{\partial \theta}\left(\mu\left[\mathrm{r} \frac{\partial}{\partial \mathrm{r}}\left(\frac{V_{\theta}}{\mathrm{r}}\right)\right]+\frac{1}{\mathrm{r}} \frac{\partial V_{\mathrm{r}}}{\partial \theta}\right) \\
& -\frac{\mu}{\mathrm{r}}\left(2\left[\frac{1}{\mathrm{r}} \frac{\partial V_{\theta}}{\partial \theta}+\frac{V_{\mathrm{r}}}{\mathrm{r}}\right]-\frac{2}{3}\left[\frac{1}{\mathrm{r}} \frac{\partial\left(\mathrm{r} V_{\mathrm{r}}\right)}{\partial \mathrm{r}}+\frac{1}{\mathrm{r}} \frac{\partial V_{\theta}}{\partial \theta}\right]\right) \\
& -\frac{\partial P}{\partial \mathrm{r}}+\rho g_{\mathrm{r}}
\end{aligned}
$$




$$
P=P_{\text {liquid }}
$$

$$
\begin{aligned}
& \rho\left(\frac{\partial V_{\theta}}{\partial t}+V_{\mathrm{r}} \frac{\partial V_{\theta}}{\partial \mathrm{r}}+\frac{V_{\theta}}{\mathrm{r}} \frac{\partial V_{\theta}}{\partial \theta}+\frac{V_{\mathrm{r}} V_{\theta}}{\mathrm{r}}\right) \\
& \quad=\frac{1}{2} \frac{\partial}{\partial r}\left(\mathrm{r}^{2} \mu\left[\mathrm{r} \frac{\partial}{\partial \mathrm{r}}\left(\frac{V_{\theta}}{\mathrm{r}}\right)+\frac{1}{\mathrm{r}} \frac{\partial V_{\mathrm{r}}}{\partial \theta}\right]\right) \\
& \quad+\frac{1}{\mathrm{r}} \frac{\partial}{\partial \theta}\left(\mu\left[\frac{2}{\mathrm{r}} \frac{\partial V_{\theta}}{\partial \theta}+\frac{2 V_{\mathrm{r}}}{\mathrm{r}}-\frac{2}{3 \mathrm{r}} \frac{\partial\left(\mathrm{r} V_{\mathrm{r}}\right)}{\partial \mathrm{r}}-\frac{2}{3 \mathrm{r}} \frac{\partial V_{\theta}}{\partial \theta}\right]\right) \\
& \quad-\frac{1}{\mathrm{r}} \frac{\partial P}{\partial \theta}+\rho g_{\theta}
\end{aligned}
$$

$$
q=0
$$

The interface movement is calculated from the liquid drain rate and the geometry. Interface conditions are equal temperature and equal velocity:

$$
\begin{aligned}
& T_{\text {gas }}=T_{\text {liquid }} \\
& V_{\theta_{\text {gas }}}=V_{\theta_{\text {liquid }}}
\end{aligned}
$$

Energy

$$
\frac{\partial \rho h}{\partial t}+\frac{\partial \rho V_{\mathrm{r}} h}{\partial \mathrm{r}}+\frac{1}{\mathrm{r}} \frac{\partial \rho V_{\theta} h}{\partial \theta}=\frac{1}{\mathrm{r}} \frac{\partial}{\partial \mathrm{r}}\left(\mathrm{r} k \frac{\partial T}{\partial \mathrm{r}}\right)+\frac{1}{\mathrm{r}} \frac{\partial}{\partial \theta}\left(\frac{k}{\mathrm{r}} \frac{\partial T}{\partial \theta}\right)
$$

Because of the numerical difficulties associated with moving boundaries, a transformation is defined which allows the transformed computational space to be invariant to the moving vapor and liquid interface. This transformation is

States:

$$
\begin{array}{ll}
\text { Gas } & P=\frac{\mathbf{R} T}{v-b}-\frac{a}{v^{2}} \\
\text { Liquid } & \begin{array}{l}
\text { NIST thermophysical properties } \\
\text { of pure fluids }
\end{array}
\end{array}
$$
graphically shown in figure 3 and is defined as follows:

$\vartheta=\theta$
$r=\frac{r}{R}$

Boundary conditions for the model are divided into three regions: tank wall, ullage pressurant gas port, and liquid drain port. The wall boundary conditions are defined as the no-slip condition, and the heat flux is specified as a function of circumference and time. These conditions are expressed as follows:

$$
\begin{aligned}
& V_{\mathrm{r}}=V_{\theta}=0 \\
& q=f(\theta, t)
\end{aligned}
$$

The ullage pressurant gas port boundary is specified at constant pressure and temperature with no heat flux:

$$
\begin{gathered}
P=P_{\text {gas }} \\
T=T_{\text {gas }} \\
q=0
\end{gathered}
$$

The liquid drain port boundary is specified as a free boundary with no circumferential velocity, at constant pressure, and with no heat flux:

$$
V_{\theta}=0
$$

$\frac{\partial}{\partial \theta}=\frac{\partial}{\partial \vartheta}-\frac{r}{R} \frac{\partial R}{\partial \vartheta} \frac{\partial}{\partial r}$

$$
\begin{aligned}
\frac{\partial^{2}}{\partial \theta^{2}}= & \frac{\partial^{2}}{\partial \vartheta^{2}}+\left(-\frac{r}{\mathrm{R}} \frac{\partial^{2} \mathrm{R}}{\partial \vartheta^{2}}+\frac{r^{2}}{\mathrm{R}^{2}} \frac{\partial \mathrm{R}}{\partial \vartheta} \frac{\partial^{2} \mathrm{R}}{\partial r \partial \vartheta}+\frac{2 r}{\mathrm{R}}\left(\frac{\partial \mathrm{R}}{\partial \vartheta}\right)^{2}\right) \frac{\partial}{\partial \mathrm{r}} \\
& -\frac{2 r}{\mathrm{R}} \frac{\partial \mathrm{R}}{\partial \vartheta} \frac{\partial^{2}}{\partial r \partial \vartheta}+\frac{r^{2}}{\mathrm{R}^{2}}\left(\frac{\partial \mathrm{R}}{\partial \vartheta}\right)^{2} \frac{\partial^{2}}{\partial r^{2}}
\end{aligned}
$$

This transformation is now applied to the model equations to obtain the applicable Navier-Stokes equations for the computational space. These equations are presented as follows:

Continuity

$$
\frac{\partial \rho}{\partial t}+\frac{1}{r \mathrm{R}} \frac{\partial\left(\rho r V_{r}\right)}{\partial r}+\frac{1}{r \mathrm{R}} \frac{\partial\left(\rho V_{\vartheta}\right)}{\partial \vartheta}-\frac{1}{\mathrm{R}^{2}} \frac{\partial \mathrm{R}}{\partial \vartheta} \frac{\partial\left(\rho V_{\vartheta}\right)}{\partial r}=0
$$


Radial momentum

$$
\begin{aligned}
& \rho\left(\frac{\partial V_{r}}{\partial t}+\left[\frac{V_{r}}{\mathrm{R}}-\frac{V_{\vartheta}}{\mathrm{R}} \frac{\partial \mathrm{R}}{\partial \vartheta}\right] \frac{\partial V_{r}}{\partial r}+\frac{V_{\vartheta}}{r \mathrm{R}} \frac{\partial V_{r}}{\partial \vartheta}-\frac{V_{\vartheta}^{2}}{r}\right) \\
& =\left(\frac{4 \mu}{3 r \mathrm{R}^{2}}-\frac{\mu}{r \mathrm{R}^{3}} \frac{\partial^{2} \mathrm{R}}{\partial \vartheta^{2}}+\frac{2 \mu}{r \mathrm{R}^{4}}\left[\frac{\partial \mathrm{R}}{\partial \vartheta}\right]^{2}\right) \frac{\partial V_{r}}{\partial r} \\
& +\left(\frac{4 \mu}{3 \mathrm{R}^{2}}+\frac{\mu}{\mathrm{R}}\left[\frac{\partial \mathrm{R}}{\partial \vartheta}\right]^{2}\right) \frac{\partial^{2} V_{r}}{\partial r^{2}}-\frac{2 \mu}{r \mathrm{R}^{3}} \frac{\partial \mathrm{R}}{\partial \vartheta} \frac{\partial^{2} V_{r}}{\partial r \vartheta} \\
& +\frac{\mu}{r^{2} \mathrm{R}^{2}} \frac{\partial^{2} V_{r}}{\partial \vartheta^{2}}-\frac{7 \mu}{3 r^{2} \mathrm{R}^{2}} \frac{\partial V_{\vartheta}}{\partial \vartheta}-\frac{2 \mu}{r \mathrm{R}^{3}} \frac{\partial \mathrm{R}}{\partial \vartheta} \frac{\partial V_{\vartheta}}{\partial r} \\
& +\frac{\mu}{3 r \mathrm{R}^{2}} \frac{\partial^{2} V_{\vartheta}}{\partial r}-\frac{\mu}{\mathrm{R}^{3}} \frac{\partial \mathrm{R}}{\partial \vartheta} \frac{\partial^{2} V_{\vartheta}}{\partial r^{2}}-\frac{4 \mu}{3 r^{2} \mathrm{R}^{2}} V_{r}-\frac{1}{\mathrm{R}} \frac{\partial P}{\partial r}+\rho g_{r}
\end{aligned}
$$

Circumferential momentum

$$
\begin{gathered}
\rho\left(\frac{\partial V_{\vartheta}}{\partial t}+\left[\frac{V_{r}}{\mathrm{R}}-\frac{V_{\vartheta}}{\mathrm{R}} \frac{\partial \mathrm{R}}{\partial \vartheta}\right] \frac{\partial V_{\vartheta}}{\partial r}+\frac{V_{\vartheta}}{r \mathrm{R}} \frac{\partial V_{\vartheta}}{\partial \vartheta}+\frac{V_{r} V_{\vartheta}}{r \mathrm{R}}\right) \\
=\frac{7 \mu}{3 r^{2} \mathrm{R}^{2}} \frac{\partial V_{r}}{\partial \vartheta}-\frac{8 \mu}{3 r \mathrm{R}^{3}} \frac{\partial \mathrm{R} \vartheta}{\partial V_{r}}+\frac{\mu}{3 r \mathrm{R}^{2}} \frac{\partial^{2} V_{r}}{\partial r \partial \vartheta} \\
-\frac{\mu}{3 r \mathrm{R}^{3}} \frac{\partial \mathrm{R}}{\partial \vartheta} \frac{\partial^{2} V_{r}}{\partial r^{2}} \\
+\left(\frac{\mu}{r \mathrm{R}^{2}}+\frac{8 \mu}{3 r \mathrm{R}^{4}}\left[\frac{\partial \mathrm{R}}{\partial \vartheta}\right]^{2}-\frac{4 \mu}{3 r \mathrm{R}^{3}} \frac{\partial^{2} \mathrm{R}}{\partial \vartheta^{2}}\right) \frac{\partial V_{\vartheta}}{\partial r} \\
+\frac{4 \mu}{3 r^{2} \mathrm{R}^{2}} \frac{\partial^{2} v_{\vartheta}}{\partial \vartheta^{2}}-\frac{8 \mu}{3 r \mathrm{R}^{3}} \frac{\partial \mathrm{R}}{\partial \vartheta} \frac{\partial^{2} V_{\vartheta}}{\partial r \partial \vartheta} \\
+\left(\frac{\mu}{\mathrm{R}^{2}}+\frac{4 \mu}{3 \mathrm{R}^{3}}\left[\frac{\partial \mathrm{R}}{\partial \vartheta}\right]^{2}\right) \frac{\partial^{2} V_{\vartheta}}{\partial r^{2}}-\frac{\mu V_{\vartheta}}{r^{2} \mathrm{R}^{2}} \\
\quad-\frac{1}{r \mathrm{R}} \frac{\partial P}{\partial \vartheta}+\frac{1}{\mathrm{R}^{2}} \frac{\partial \mathrm{R}}{\partial \vartheta} \frac{\partial P}{\partial r}+\rho g_{\vartheta}
\end{gathered}
$$

Energy

$$
\begin{aligned}
\frac{\partial \rho h}{\partial t} & +\frac{1}{\mathrm{R}} \frac{\partial \rho V_{r} h}{\partial r}+\frac{1}{r \mathrm{R}} \frac{\partial \rho V_{\theta} h}{\partial \vartheta}-\frac{1}{\mathrm{R}^{2}} \frac{\partial \mathrm{R}}{\partial \vartheta} \frac{\partial \rho V_{\theta} h}{\partial r} \\
& =\left(\frac{2 k}{r \mathrm{R}^{4}}\left[\frac{\partial \mathrm{R}}{\partial \vartheta}\right]^{2}-\frac{k}{r \mathrm{R}^{3}} \frac{\partial^{2} \mathrm{R}}{\partial \vartheta^{2}}\right) \frac{\partial T}{\partial \mathrm{r}}+\frac{k}{r \mathrm{R}^{2}} \frac{\partial}{\partial r}\left(r \frac{\partial T}{\partial r}\right) \\
& +\frac{k}{\mathrm{R}^{4}}\left[\frac{\partial \mathrm{R}}{\partial \vartheta}\right]^{2} \frac{\partial^{2} T}{\partial r^{2}}-\frac{2 k}{r \mathrm{R}^{3}} \frac{\partial \mathrm{R}}{\partial \vartheta} \frac{\partial^{2} T}{\partial r \partial \vartheta}+\frac{k}{r^{2} \mathrm{R}^{2}} \frac{\partial^{2} T}{\partial \vartheta^{2}}
\end{aligned}
$$

Gas and liquid states remain unchanged.

Boundary conditions at tank wall

$$
\begin{aligned}
& V_{r}=V_{\vartheta}=0 \\
& q=f(\vartheta, t)
\end{aligned}
$$

The boundary conditions at the ullage pressurant and liquid drain ports remain unchanged. Interface conditions also remain unchanged.

Note that the transformed equations reduce to the standard Navier-Stokes equations for cylindrical coordinates when

$$
R=\text { constant } \quad \text { and } \quad \frac{\partial R}{\partial \vartheta}=\frac{\partial^{2} R}{\partial \vartheta^{2}}=0
$$

\section{Numerical Method}

Finite-difference equations were specifically developed for this model using a volume integral method technique which is discussed in lecture notes by Lick. ${ }^{8}$ The development of the continuity and energy equations is purely explicit, time-marching algorithms. The development of the momentum equations is longitudinally implicit, transversely explicit, time-marching algorithms. Longitudinally implicit means, for example, that in solving for $V_{r}$ in the radial momentum equation all terms that are discretized in the radial direction are implicit. Conversely, terms that are discretized in the theta direction are explicit (reverse for the theta momentum equation).

The longitudinally implicit approach to the momentum equations was chosen to increase the stability of the solution to the nonlinear Navier-Stokes equations. This approach improved Reynolds cell instability associated with purely explicit time-marching techniques. The finitedifference equations for the transformed Navier-Stokes equations are second-order accurate in space and firstorder accurate in time and are presented as follows:

\section{Continuity}

$$
\begin{aligned}
\rho_{\mathrm{i}, \mathrm{j}}^{\mathrm{n}+1} & =\rho_{\mathrm{i}, \mathrm{j}}^{\mathrm{n}} \\
& +\left(\frac{\mathrm{r}_{\mathrm{i}-}}{\mathrm{r}_{\mathrm{i}}} \mathrm{FI}_{\mathrm{i}-, \mathrm{j}}^{1}-\frac{\mathrm{r}_{\mathrm{i}+}}{\mathrm{r}_{\mathrm{i}}} \mathrm{FI}_{\mathrm{i}+, \mathrm{j}}^{1}+\mathrm{FI}_{\mathrm{i}-, \mathrm{j}}^{2}-\mathrm{FI}_{\mathrm{i}+, \mathrm{j}}^{2}\right) \frac{\Delta t}{\Delta r} \\
& +\frac{1}{\mathrm{R}_{\mathrm{i}, \mathrm{j}}^{\mathrm{n}}}\left(\mathrm{FJ}_{\mathrm{i}, \mathrm{j}-}-\mathrm{FJ}_{\mathrm{i}, \mathrm{j}+}\right) \frac{\Delta t}{\Delta r}
\end{aligned}
$$


where

$$
\begin{aligned}
& F_{i+, j}^{I}=\left(\frac{\rho V_{r}}{R}\right)_{i+, j}^{n} \\
& F_{i+, j}^{2}=\left(\frac{\rho V_{\vartheta}}{R^{2}} \frac{\partial R}{\partial \vartheta}\right)_{i+, j}^{n} \\
& F_{i, j+}=\left(\frac{\rho V_{\vartheta}}{r}\right)_{i, j+}^{n}
\end{aligned}
$$

Radial momentum

$$
\begin{aligned}
& V_{r_{i, j}}^{n+1}=V_{r_{i, j}}^{n}+\left(-\frac{V_{r_{i, j}}^{n}}{R_{j}^{n}}+\frac{V_{\vartheta_{i, j}}^{n}}{R_{j}^{n 2}} \frac{\partial R_{j}^{n}}{\partial \vartheta}+\frac{\mu_{i, j}}{\rho_{i, j}^{n} R_{j}^{n 3}}\right. \\
& \left.\left(\frac{2}{r_{i, j} \mathrm{R}_{\mathrm{j}}^{\mathrm{n}}}\left(\frac{\partial \mathrm{R}_{\mathrm{j}}^{\mathrm{n}}}{\partial \vartheta}\right)^{2}-\frac{1}{r_{\mathrm{i}}} \frac{\partial^{2} \mathrm{R}_{\mathrm{j}}^{\mathrm{n}}}{\partial \vartheta^{2}}\right)\right) \\
& \left(V_{r_{i+1, j}+1}^{n+1}-V_{r_{i-1, j}+1}\right) \frac{\Delta t}{2 \Delta r}+\left(\frac{4 \mu_{i, j}}{3 \rho_{i, j}^{n} R_{j}^{n 2}}+\frac{\mu_{i, j}}{\rho_{i, j}^{n} R_{j}^{n 4}}\left(\frac{\partial R_{j}^{n}}{\partial \vartheta}\right)^{2}\right) \\
& \left(\frac{r_{i+, j}}{r_{i, j}} V_{r_{i+1, j}+1}^{n}-2 V_{r_{i, j}}^{n+1}+\frac{r_{i-, j}}{r_{i, j}} V_{r_{i-1, j}}^{n+1}\right) \frac{\Delta t}{\Delta r^{2}} \\
& -\left(\frac{v_{r_{i, j}}^{n}}{r_{i, j} \mathbf{R}_{j}^{n}}+\frac{7 \mu_{i, j}}{3 \rho_{i, j}^{n} r_{i, j}^{2} \mathbf{R}_{j}^{n 2}}\right)\left(v_{\theta_{i, j+}}^{n}-v_{\theta_{i, j}}^{n}\right) \frac{\Delta t}{\Delta \vartheta} \\
& +\frac{\mu_{i, j}}{\rho_{i, j}^{n} r_{1, j}^{2} R_{j}^{n 2}}\left(v_{r_{i, j+1}^{n+1}}^{n}-2 V_{r_{i, j}+1}^{n+1}+v_{r_{i, j-1}+1}\right) \frac{\Delta t}{\Delta \vartheta^{2}} \\
& +\frac{4 \mu_{i, j}}{3 p_{i, j}^{n} r_{i, j} R_{j}^{n 4}} \frac{\partial R_{j}^{n}}{\partial \vartheta}\left(v_{\vartheta_{i+, j}}^{n}-V_{\theta_{i-, j}}^{n}\right) \frac{\Delta t}{\Delta r} \\
& +\frac{\mu_{i, j}}{3 \rho_{i, j}^{n} R_{j}^{n 3}} \frac{\partial R_{j}^{n}}{\partial \vartheta}\left(V_{\theta_{i+1, j}^{n}}^{n}-2 V_{\theta_{i, j}}^{n}+V_{\theta_{i-1, j}}^{n}\right) \frac{\Delta t}{\Delta r^{2}} \\
& -\frac{4 \mu_{i, j}}{3 p_{i, j}^{n} r_{i, j}^{2} R_{j}^{n 4}} V_{r_{i, j}}^{n+1} \Delta t+\frac{V_{\theta_{i, j}}^{n}}{r_{i} R_{j}^{n}} \Delta t+g_{r_{i, j}} \Delta t \\
& -\frac{\left(P_{\mathrm{i}+, \mathrm{j}}^{\mathrm{n}+1}-P_{\mathrm{i}-\mathrm{j}}^{\mathrm{n}+1}\right)}{\mathrm{R}_{\mathrm{j}}^{\mathrm{n}} \mathrm{\rho}_{\mathrm{i}, \mathrm{j}}^{\mathrm{n}+1}} \frac{\Delta t}{\Delta r}
\end{aligned}
$$

where the pressure term, $P^{n+1}$, in the radial momentum equation is approximated to first order in time by combining the continuity equation with an ideal equation of state. The result is as follows:

$$
\begin{aligned}
P_{\mathrm{i}, \mathrm{j}}^{\mathrm{n}+1} & =P_{\mathrm{i}, \mathrm{j}}^{\mathrm{n}}+\Delta P_{\mathrm{i}, \mathrm{j}}^{\mathrm{n} \rightarrow \mathrm{n}+1} \\
P_{\mathrm{i}+\mathrm{j}}^{\mathrm{n}+1} & =P_{\mathrm{i}+, \mathrm{j}}^{\mathrm{n}} \\
& +\frac{\rho_{\mathrm{i}+, \mathrm{j}}^{\mathrm{n}} \mathrm{RT}_{\mathrm{i}+, \mathrm{j}}}{\mathrm{R}_{\mathrm{j}}^{\mathrm{n}}}\left(\left(V_{r_{\mathrm{i}, \mathrm{j}}^{\mathrm{n}}}^{\mathrm{n}}-V_{r_{\mathrm{i}+1, \mathrm{j}}^{\mathrm{n}}}^{\mathrm{n}}\right) \frac{\Delta t}{\Delta r}\right. \\
& \left.+\left(V_{\vartheta_{\mathrm{i}, \mathrm{j}-1}}^{\mathrm{n}}-V_{\vartheta_{\mathrm{i}, \mathrm{j}}}^{\mathrm{n}}\right) \frac{\Delta t}{r \Delta \vartheta}\right)
\end{aligned}
$$

Circumferential momentum

$$
\begin{aligned}
& V_{\vartheta_{i, j}}^{\mathrm{n}+1}=V_{\vartheta_{i, j}}^{\mathrm{n}}-\frac{V_{\vartheta_{i, j}}^{\mathrm{n}}}{2 r_{\mathrm{i}, \mathrm{j}} \mathrm{R}_{\mathrm{j}}^{\mathrm{n}}}\left(V_{\vartheta_{\mathrm{i}, \mathrm{j}+1}^{\mathrm{n}+1}}-V_{\theta_{\mathrm{i}, j-1}}^{\mathrm{n}+1}\right) \frac{\Delta t}{\Delta \vartheta} \\
& +\frac{4 \mu_{i, j}}{3 \rho_{i, j}^{n} r_{i, j}^{2} R_{j}^{n 2}}\left(v_{\vartheta_{i, j+1}}^{n+1}-2 v_{\vartheta_{i, j}}^{n+1}+v_{\theta_{i, j-1}}^{n+1}\right) \frac{\Delta t}{\Delta \vartheta^{2}} \\
& +\frac{7 \mu_{i, j}}{3 \rho_{i, j}^{n} r_{i, j}^{2} R_{j}^{n 2}}\left(V_{r_{i, j+}}^{n}-V_{r_{i, j}}^{n}\right) \frac{\Delta t}{\Delta \vartheta} \\
& +\frac{8 \mu_{\mathrm{i}, \mathrm{j}}}{3 \mathrm{p}_{\mathrm{i}, \mathrm{j}}^{\mathrm{n}} r_{\mathrm{i}, \mathrm{j}} \mathrm{R}_{\mathrm{j}}^{\mathrm{n} 3}} \frac{\partial \mathrm{R}_{\mathrm{j}}^{\mathrm{n}}}{\partial \vartheta}\left(v_{r_{\mathrm{i}+, \mathrm{j}}}^{\mathrm{n}}-v_{r_{\mathrm{i}-\mathrm{j}}}^{\mathrm{n}}\right) \frac{\Delta t}{\Delta r} \\
& -\frac{\mu_{i, j}}{3 p_{i, j}^{n} r_{i, j} R_{j}^{n 3}} \frac{\partial R_{j}^{n}}{\partial \vartheta}\left(V_{r_{i+l, j}}^{n}-2 V_{r_{i, j}}^{n}+V_{r_{i-1, j}}^{n}\right) \frac{\Delta t}{\Delta r^{2}} \\
& +\left(\frac{\mu_{i, j}}{\rho_{i, j}^{n} R_{j}^{n 2}}\left(\frac{1}{r_{i, j}}+\frac{8}{3 r_{i, j} R_{j}^{n 2}}\left(\frac{\partial R_{j}^{n}}{\partial \vartheta}\right)^{2}-\frac{4}{3 r_{i} R_{j}^{n}} \frac{\partial^{2} R_{j}^{n}}{\partial \vartheta^{2}}\right)\right. \\
& \left.-\frac{V_{r_{i, j}}^{n}}{R_{j}^{n}}+\frac{V_{\theta_{i, j}}^{n}}{R_{j}^{n}} \frac{\partial R_{j}^{n}}{\partial \vartheta}\right)\left(V_{\theta_{i+1, j}}^{n}-V_{\theta_{i-1, j}}^{n}\right) \frac{\Delta t}{2 \Delta r} \\
& +\frac{\mu_{i, j}}{\rho_{i, j}^{n} R_{j}^{n 2}}\left(1+\frac{4}{3 R_{j}^{n 2}}\left(\frac{\partial R_{j}^{n}}{\partial \vartheta}\right)^{2}\right) \\
& \left(v_{\theta_{i+1, j}}^{\mathrm{n}}-2 v_{\theta_{i, j}}^{\mathrm{n}}+v_{\theta_{i-1, j}}^{\mathrm{n}}\right) \frac{\Delta t}{\Delta r^{2}}
\end{aligned}
$$




$$
\begin{aligned}
& -\left(\frac{\mu_{i, j}}{\rho_{i, j}^{n} r_{1}^{2} R_{j}^{n 2}}+\frac{V_{r_{i, j}}^{n}}{r_{i} R_{j}^{n}}\right) V_{\vartheta_{i, j}^{n+1}}^{n} \Delta t \\
& +\frac{1}{\rho_{\mathrm{i}, \mathrm{j}}^{\mathrm{n}} \mathrm{R}_{\mathrm{j}}^{\mathrm{n} 2}} \frac{\partial \mathrm{R}_{\mathrm{j}}^{\mathrm{n}}}{\partial \vartheta}\left(P_{\mathrm{i}+, \mathrm{j}}^{\mathrm{n}}-P_{\mathrm{i}+, \mathrm{j}}^{\mathrm{n}}\right) \frac{\Delta t}{\Delta r}+g_{\vartheta_{\mathrm{i}, \mathrm{j}}} \Delta t \\
& -\frac{\left(P_{\mathrm{i}, \mathrm{j}+}^{\mathrm{n}}-P_{\mathrm{i}, \mathrm{j}-}^{\mathrm{n}}\right)}{\rho_{\mathrm{i}, \mathrm{j}}^{\mathrm{n}} r_{\mathrm{i}} \mathrm{R}_{\mathrm{j}}^{\mathrm{n}}} \frac{\Delta t}{\Delta \vartheta} \\
& -\frac{R T_{i+, j}}{\rho_{i, j}^{n} r_{1}^{2} R_{j}^{n 2}}\left(\rho_{i, j+}^{n}\left(V_{\vartheta_{i, j}}^{n+1}-V_{\vartheta_{i, j+1}}^{n+1}\right)\right. \\
& \left.-\rho_{i, j-}^{n}\left(V_{\vartheta_{i, j-1}}^{n+1}-V_{\vartheta_{i, j}}^{n+1}\right)\right) \frac{\Delta t^{2}}{\Delta \vartheta^{2}} \\
& -\frac{\mathbf{R T}_{\mathrm{i}+, \mathrm{j}}}{\rho_{\mathrm{i}, \mathrm{j}}^{\mathrm{n}} r_{1}^{2} \mathrm{R}_{\mathrm{j}}^{\mathrm{n} 2}}\left(\rho_{\mathrm{i}, \mathrm{j}+}^{\mathrm{n}}\left(\frac{r_{\mathrm{i}-}}{r_{\mathrm{i}}} V_{r_{\mathrm{i}-, \mathrm{j}}}^{\mathrm{n}}-\frac{r_{\mathrm{i}+}}{r_{\mathrm{i}}} V_{r_{\mathrm{i}+\mathrm{j}+}}^{\mathrm{n}}\right)\right. \\
& \left.-\rho_{\mathrm{i}, \mathrm{j}-}^{\mathrm{n}}\left(\frac{r_{\mathrm{i}-}}{r_{\mathrm{i}}} V_{r_{\mathrm{i}-\mathrm{j}-\mathrm{j}}}^{\mathrm{n}}-\frac{r_{\mathrm{i}+}}{r_{\mathrm{i}}} V_{r_{\mathrm{i}+\mathrm{j}}}^{\mathrm{n}}\right)\right) \frac{\Delta t^{2}}{\Delta r \Delta \vartheta}
\end{aligned}
$$

Energy

$$
h_{\mathrm{i}, \mathrm{j}}^{\mathrm{n}+1}=h_{\mathrm{i}, \mathrm{j}}^{\mathrm{n}}+\frac{1}{r_{\mathrm{i}}}\left(\mathrm{FI}_{\mathrm{i}+, \mathrm{j}}-\mathrm{FI}_{\mathrm{i}-, \mathrm{j}}\right)+\left(\mathrm{FJ}_{\mathrm{i}, \mathrm{j}+}-\mathrm{FJ}_{\mathrm{i}, \mathrm{j}}\right)
$$

where

$$
\begin{aligned}
& \mathrm{FI}_{\mathrm{i}+\mathrm{j}}= \\
& \left(\frac{-r_{i+} V_{r_{i+, j}}^{n}}{R_{j}^{n}}+\frac{r_{i+} V_{r_{i+j}}^{n}}{R_{j}^{n 2}} \frac{\partial R_{j}^{n}}{\partial \vartheta}\right) \rho_{i+, j}^{n} h_{i+, j}^{n} \\
& +k_{\mathrm{i}+, \mathrm{j}}^{\mathrm{n}}\left[\frac{1}{\mathrm{R}_{\mathrm{j}}^{\mathrm{n} 3}} \frac{\partial^{2} \mathrm{R}_{\mathrm{j}}^{\mathrm{n}}}{\partial \vartheta^{2}}-\frac{2}{\mathrm{R}_{\mathrm{j}}^{\mathrm{n}}}\left(\frac{\partial \mathrm{R}_{\mathrm{j}}^{\mathrm{n}}}{\partial \vartheta^{2}}\right)^{2}\right] T_{\mathrm{i}+, \mathrm{j}}^{\mathrm{n}}
\end{aligned}
$$

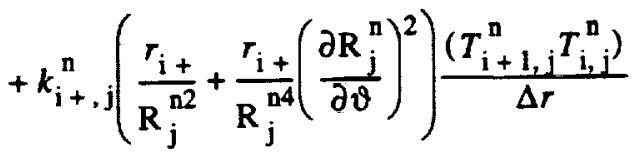

$$
\begin{aligned}
& F J_{i, j+}=k_{i, j+}^{n} \frac{1}{r_{i}^{2} R_{j+}^{n 2}} \frac{\left(T_{i, j+1}^{n}-T_{i, j}^{n}\right)}{\Delta \vartheta}-\frac{V_{i+, j}^{n}}{r_{i} R_{j+}^{n}} \rho_{i, j+}^{n} h_{i, j+}^{n}
\end{aligned}
$$

The boundary and interface finite-difference equations are derived directly from the volume integral method technique and are very similar to these equations. Because of the similarity, they are not shown. These equations form a complete set of time-marching, finite-difference equations. The first step in the numerical procedure is to solve the momentum equations for the velocities at the next time step, $V_{r}^{n+1}$ and $V_{\vartheta}^{n+1}$. The formulation of the momentum difference equations is such that a tridiagonal matrix is solved for each ray of radial velocities. In addition, a tridiagonal matrix is solved for each circlet of circumferential velocities. The average velocity over the time step, $V^{\text {avg }}=\left(V^{n+1}+V^{n}\right) / 2$, is then used in the continuity and energy equations to obtain density and temperature for the next time step, $\rho_{i, j}^{n+1}$ and $T_{i, j}^{n+1}$. The pressure, $P_{i, j}^{n+1}$, is then calculated from the state equation as a function of $\rho_{\mathrm{i}, \mathrm{j}}^{\mathrm{i}}$ and $T_{\mathrm{i}, \mathrm{j}}^{\mathrm{n}+\mathrm{l}}$.

A staggered grid was used to develop the circumferential and radial velocities (that is, velocity nodes centered between pressure nodes). The staggered grid was selected to avoid special consideration of the no-slip radial boundary condition. This special consideration arises because longitudinal pressure disturbances or acoustical waves traveling in the radial direction are not correctly modeled in a standard grid by assuming the wall velocity to be zero. The fluid molecules in the vicinity near the wall have velocity when disturbed by radial longitudinal acoustical waves. Thus, specifying a zero velocity for a wall boundary node does not represent the total average velocity for the boundary node when using a standard grid. This difficulty is avoided by using a staggered grid and a no-flux boundary condition at the wall because the radial velocities are not calculated at the boundary in the staggered grid but are calculated at a location of $\Delta r / 2$ from the boundary. In addition to the staggered grid, variable node spacing was incorporated to model the tank wall boundary layer with more densely populated nodes.

The stiffness often associated with the Navier-Stokes equations can present numerical difficulties. Stiffness is a term which characterizes a system which has two vastly different time scales. In this problem, the stiffness results from the disparity between the numerical time step associated with the speed of sound in the fluid, of the order of $0.00001 \mathrm{sec}$, and the drain time of the tank, of the order of $1000 \mathrm{sec}$. The numerical solution would require an order of $100,000,000$ time steps. One method of dealing with the difficulties associated with stiffness is to adjust the characteristic time scales of the variables. 9.10 The stiffness in this problem is reduced by adjusting the Mach number of the fluid by artificially decreasing the speed of sound in the computation. The Mach number of the flow within the cryogenic tank is expected to be of the order of 0.0001 which is nearly incompressible. Because compressible flows with Mach numbers of 0.1 or less can be approximated as incompressible flows with less than 1 percent error ", it is reasonable to expect that the Mach number of this problem can be increased from 0.0001 to 
0.1 with less than 1 percent error. The Mach number is increased in the computation by scaling the pressure variable as it relates to the speed of sound through the relation,

$$
c^{2}=\left(\frac{\partial P}{\partial \rho}\right)_{s}
$$

The numerical time step associated with the scaled pressure variable can be increased to the order of $0.01 \mathrm{sec}$, thus reducing the required time steps for solution to 100,000 .

\section{Results and Discussion}

These numerical calculations were conducted on small grids $(0.01-\mathrm{m}$ radius with 280 nodes and $0.05-\mathrm{m}$ radius with 345 nodes) to save computer resources during model development. Modeling of the generic research cryogenic tank will require a larger grid $(0.75$-m radius with approximately 5,000 to 10,000 nodes). This grid will substantially increase computer resources. Full-scale calculations were considered an unwarranted use of computer resources because testing of the generic research cryogenic tank has not begun. Data for model comparison are currently not available.

Figures 4 and 5 show the numerical effect of scaling the Mach number. These figures present time histories of velocity at the center of the gas region (fig. 4) and at the center of the liquid region (fig. 5) for various Mach scaling factors (MSF). The calculations were performed assuming a tank radius of $0.01 \mathrm{~m}$ with a 10 - by 28 -node grid. Initially, the gas and liquid regions are at rest. The model is begun by applying a constant impulsive drain rate velocity at the gas and liquid interface. These figures represent the time response to an impulsively applied drain rate. Excellent agreement for Mach scaling factors up to 100 and fair agreement for Mach scaling factors up to 1000 are also shown. The time step required for stability in these calculations were 1.e-9 sec (MSF = 1), 1.e-7 sec (MSF = 100), and 1.e-6 sec (MSF = 1000). Thus by using a Mach scaling factor of 1000 , computational time is decreased by three orders of magnitude in this test case.

Figures 4 and 5 also show the physical modeling effect of scaling the Mach numbers. Note that the time axis is a ratio of the Mach scaling factor. Thus when an impulsive disturbance is applied to the model, scaling the Mach number increases the time required for the modeled fluid to respond to the disturbance. The final effect of the disturbance, or the velocities, remain unchanged. In this model, the interest is in fluid characteristics which occur over large time scales (minutes). As a result, characteristic responses which occur in time scales of microseconds can be increased to seconds as long as they do not affect the final result.
Figures 6 and 7 show the numerical results of applying a first-order correction to the pressure term

$$
\begin{aligned}
P_{\mathrm{i}+, \mathrm{j}}^{\mathrm{n}+1}= & P_{\mathrm{i}+, \mathrm{j}}^{\mathrm{n}+1}+\frac{\rho_{\mathrm{i}+, \mathrm{j}}^{\mathrm{n}} \mathbf{R} \mathrm{T}_{\mathrm{i}+, \mathrm{j}}}{\mathrm{R}_{\mathrm{j}}^{\mathrm{n}}} \\
& \left(\left(V_{r_{\mathrm{i}, \mathrm{j}}}^{\mathrm{n}}-V_{r_{\mathrm{i}+\mathrm{l}, \mathrm{j}}}^{\mathrm{n}}\right) \frac{\Delta t}{\Delta r}+\left(V_{\theta_{\mathrm{i}, \mathrm{j}-1}}^{\mathrm{n}}-v_{\vartheta_{\mathrm{i}, \mathrm{j}}}^{\mathrm{n}}\right) \frac{\Delta t}{r \Delta \vartheta}\right) \\
P_{\mathrm{i}, \mathrm{j}+}^{\mathrm{n}+1}= & P_{\mathrm{i}, \mathrm{j}+}^{\mathrm{n}}+\frac{\rho_{\mathrm{i}, \mathrm{j}+}^{\mathrm{n}} \mathbf{R T} \mathrm{i}+, \mathrm{j}}{\mathrm{R}_{\mathrm{j} \mathrm{p}}^{\mathrm{n}}} \\
& \left(\left(V_{r_{\mathrm{i}-\mathrm{j}+}^{\mathrm{n}}}-\mathrm{V}_{r_{\mathrm{i}+, \mathrm{j}}}^{\mathrm{n}}\right) \frac{\Delta t}{\Delta r}+\left(V_{\theta_{\mathrm{i}, \mathrm{j}}}^{\mathrm{n}}-V_{\theta_{\mathrm{i}, \mathrm{j}+1}^{\mathrm{n}}}^{\mathrm{n}}\right) \frac{\Delta t}{r \Delta \vartheta}\right.
\end{aligned}
$$

in the momentum equations for the ullage gas and liquid regions, respectively. In these figures, $\mathbf{R} / \mathbf{R}^{*}$ is the ratio of the fluid constant used in the numerical calculations to the ideal fluid constant. Thus, an $\mathbf{R} / \mathbf{R}^{*}=0$ would imply no first-order correction. An $\mathbf{R}^{*} \mathbf{R}^{*}=1$ would imply an ideal first-order correction. The deviation is defined as the maximum velocity difference between a particular test case and a standard test case calculated with a time step of 1.e$6 \mathrm{sec}$ and $\mathbf{R} / \mathbf{R}^{*}$ set at zero.

These calculations were performed assuming a tank radius of $0.01 \mathrm{~m}$ with a 10 - by 28 -node grid and a Mach scaling factor of 100 . As expected, the first-order correction term increased the stability of the program and allowed larger time steps in the calculations. However, the large deviations with increasing $\mathbf{R} / \mathbf{R}^{*}$ for the liquid (17 percent at $\mathbf{R} / \mathbf{R}^{*}=1, \Delta t=0.5 \mathrm{e}-5$ ) were unanticipated. The large difference in deviation between the gas and liquid regions may result from the difference in Reynolds cell number. The average Reynolds cell number in the gas region is 0.65 compared to 8.5 in the liquid region. Reynolds cell numbers greater than 2 are known to cause instabilities in the finite-difference technique. An $\boldsymbol{R} / \boldsymbol{R}^{*}=$ 0.25 appears to be the optimal value for this test case. As a result, the computational time could be reduced by half (by doubling time step) with a loss in numerical accuracy of less than 3 percent in the gas region and 10 percent in the liquid region.

Figures 8(a), (b), and (c) show the effect of the moving interface. These figures present velocity vectors at twothirds, one-half, and one-third of the gas and liquid interface heights. The calculations were conducted on one side of the tank as symmetry is assumed, a tank radius of 0.05 $\mathrm{m}$, a 15- by 23-node grid, a Mach scaling factor of 100 , an $\mathbf{R} / \mathbf{R}^{*}$ set at 0.25 , and a drain rate of $0.002 \mathrm{~m}^{3} / \mathrm{sec}$. 


\section{Concluding Remarks}

A computational fluid dynamic model has been developed to model a cryogenic fluid tank while subjected to rapid drain rate and high heat flux. The model incorporates a moving interface, a transient hybrid explicit-implicit finite-difference technique, a Mach scaling factor, and a first-order correction to the pressure term in the momentum equations. The Mach scaling factor decreases the computational time of the model without a significant loss of accuracy. The first-order correction to the pressure term increases stability and decreases computational time with some loss of accuracy.

The generic research cryogenic tank which this model was developed to simulate has met with budgetary constrains, and test data for verification of the model are unavailable. Comparison of the model with test data will be the subject of future efforts.

\section{References}

'Fenn D.B., Acker L.W. and Algranti J.S., Flight Operation of a Pump-Fed, Liquid Hydrogen Fuel System, NASA TM-252, Apr. 1960.

2Brewer, G.D., Hydrogen Aircrafi Technology, CRC Press, Inc., Boca Raton, Florida, 1991.

${ }^{3}$ Hellwig, W., Clifft, G., Seidensticker, D., Dayton, K., and Wilson, T., (Draft) Test Report for McDonnell Douglas NASP Task D (U), Repon X30MD92007, McDonnell Douglas Aerospace, St. Louis, Missouri, Dec. 1992.
'Cope, K.W. and Thomdyke, P., Test Report for GD Task D Tank Cryogenic Test, Report X30GD92003, General Dynamics, San Diego, California, Mar. 1992.

SStephens, C.A., Hanna, G.J. and Gong, L., ThermalFluid Analysis of the Fill and Drain Operations of a Cryogenic Fuel Tank, NASA TM-104273, Dec. 1993.

${ }^{6}$ Anderson, J.D., Jr., Hypersonic and High Temperature Gas Dynamics, McGraw Hill, New York, 1989.

${ }^{7}$ McCarty, R.D., Arp, V., and Friend, D.G., NIST Thermophysical Properties of Pure Fluids, Version 3.0, Users Guide: NIST Standard Reference Database 12, National Institute of Standards and Technology, Gaithersburg, Maryland, 1992.

"Lick, WJ., "Difference Equations from Differential Equations," Engineering Ser., vol. 41, Springer-Verlag. New York, 1989.

"Chorin, AJ., "A Numerical Method for Solving Incompressible Viscous Flow Problems," $J$ of Computational Physics, vol. 2, 1967, pp. 12-26.

${ }^{10}$ Bussing, T.R.A. and Murman, E.M., "Finite-Volume Method for the Calculation of Compressible Chemically Reacting Flows," AIAA Journal, vol. 26, no. 9, Sept. 1988, pp. 1070-1078.

"Schlichting, H., Boundary-Layer Theory, McGraw Hill, New York, 1979.

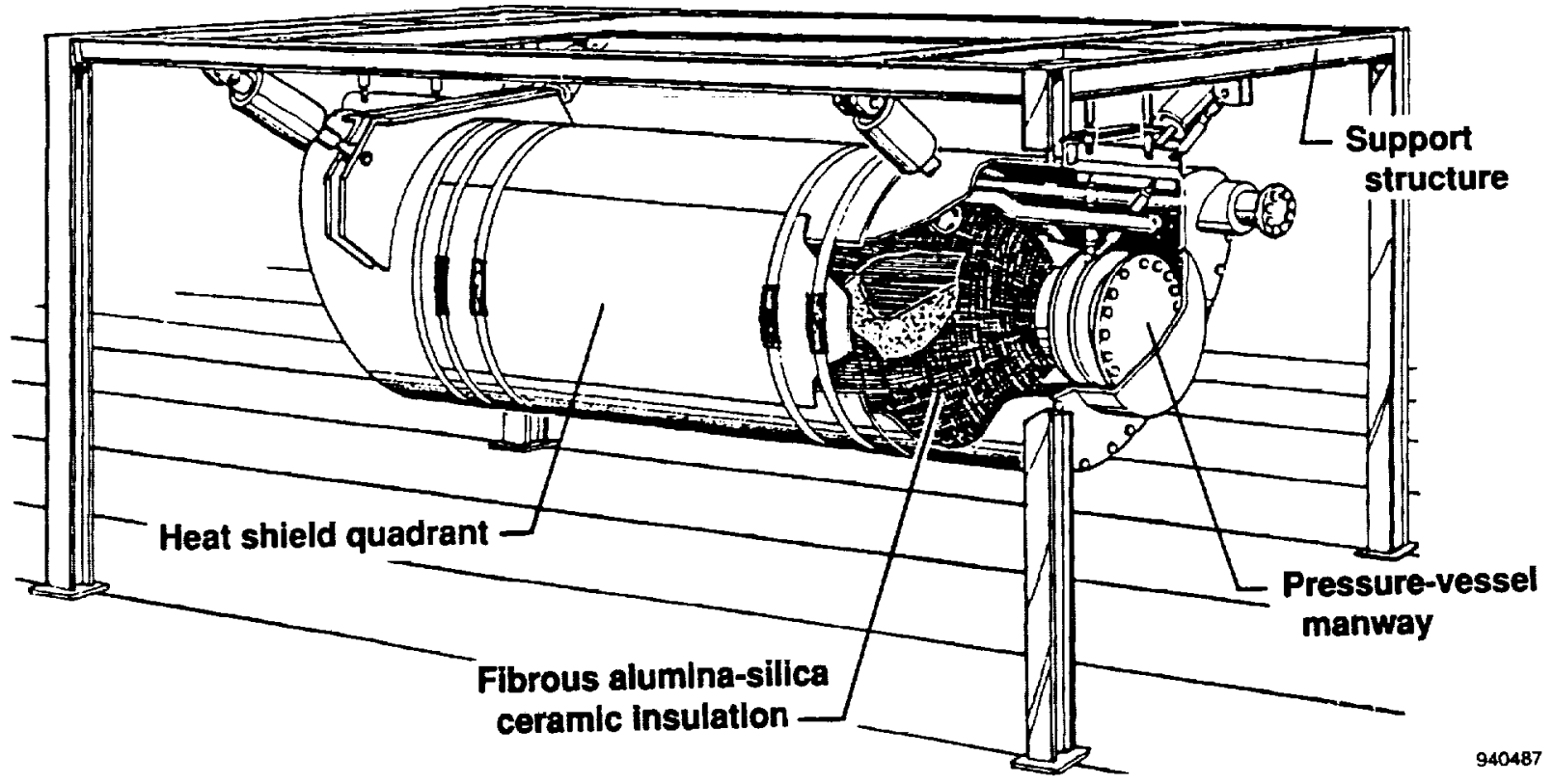

Figure 1. The generic research cryogenic tank. 
Pressurization gas: Specify incoming

gas pressure, $P(t)$, and temperature, $T(t)$

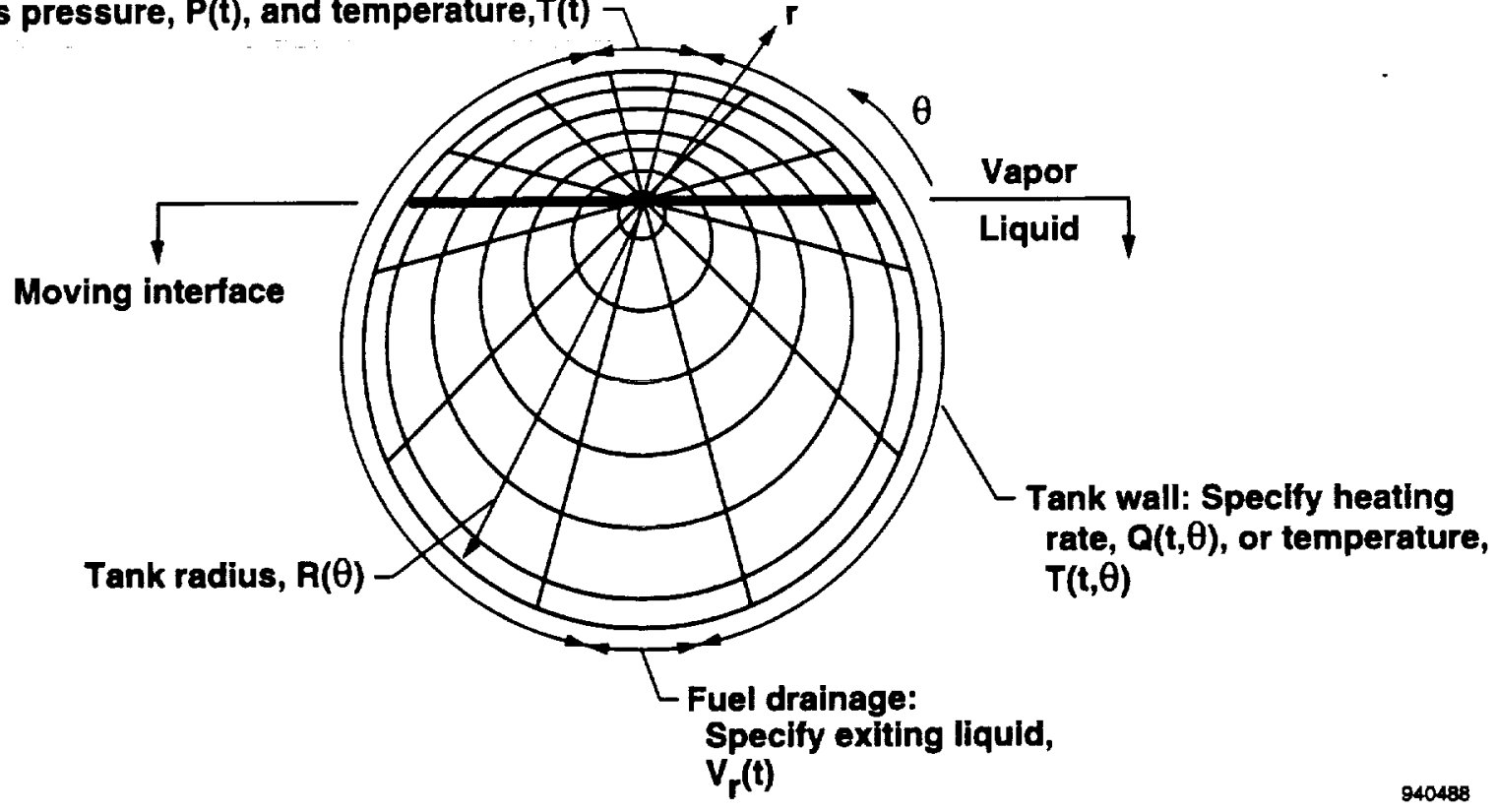

Figure 2. Modeled cross-sectional area.

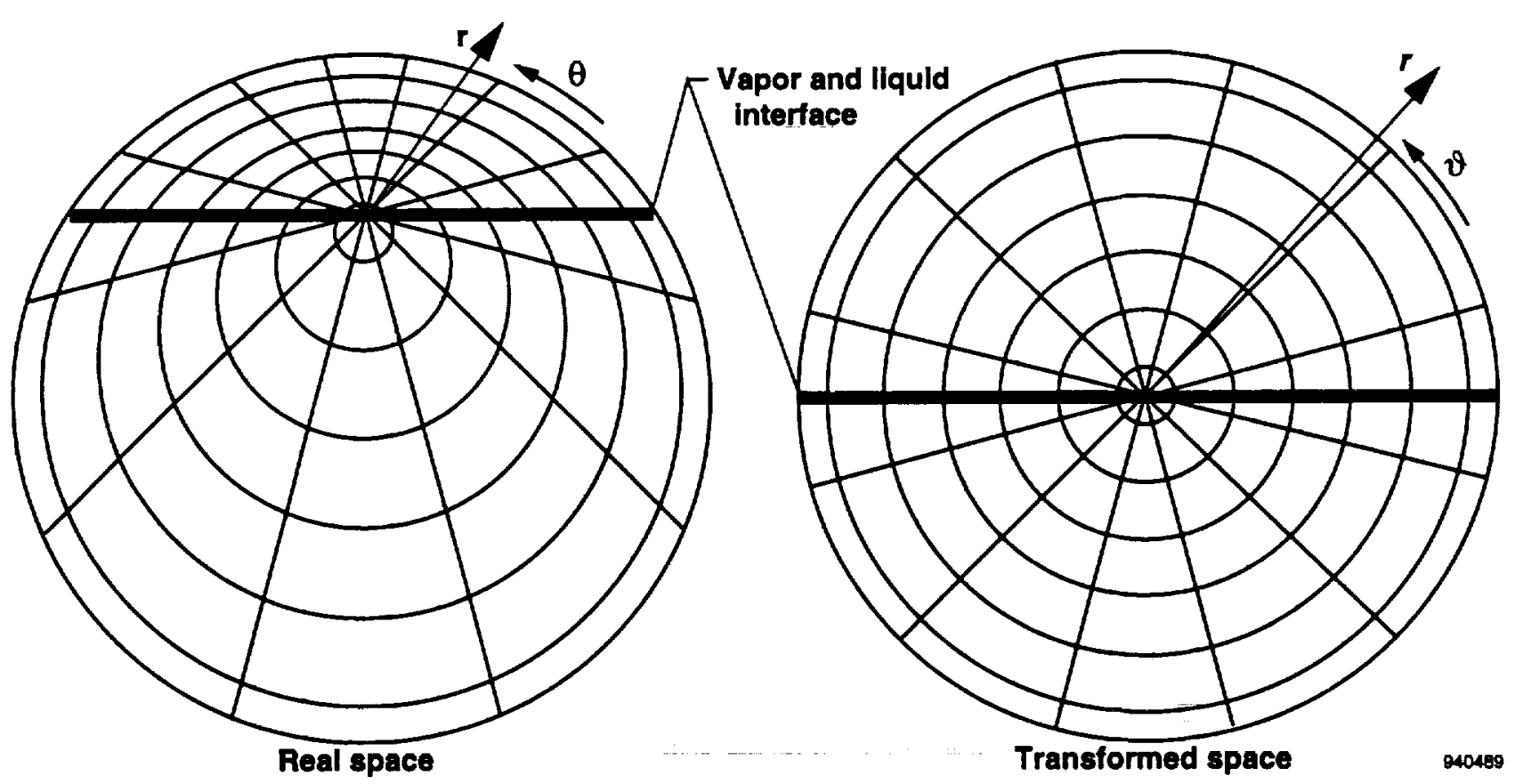

Figure 3. Coordinate transformation relative to the vapor and liquid interface. 


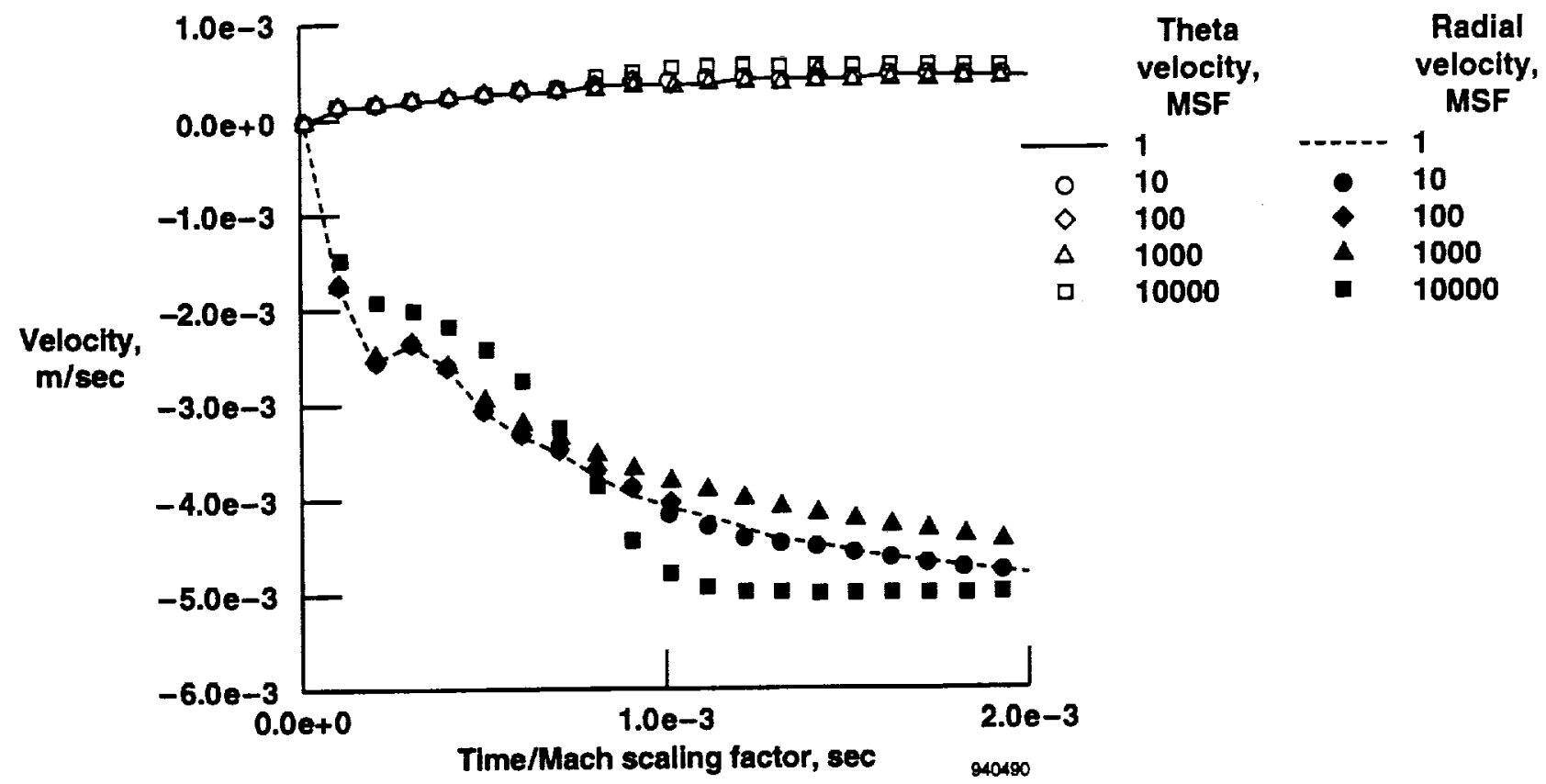

Figure 4. Numerical effect of scaling the Mach number in the gas region.

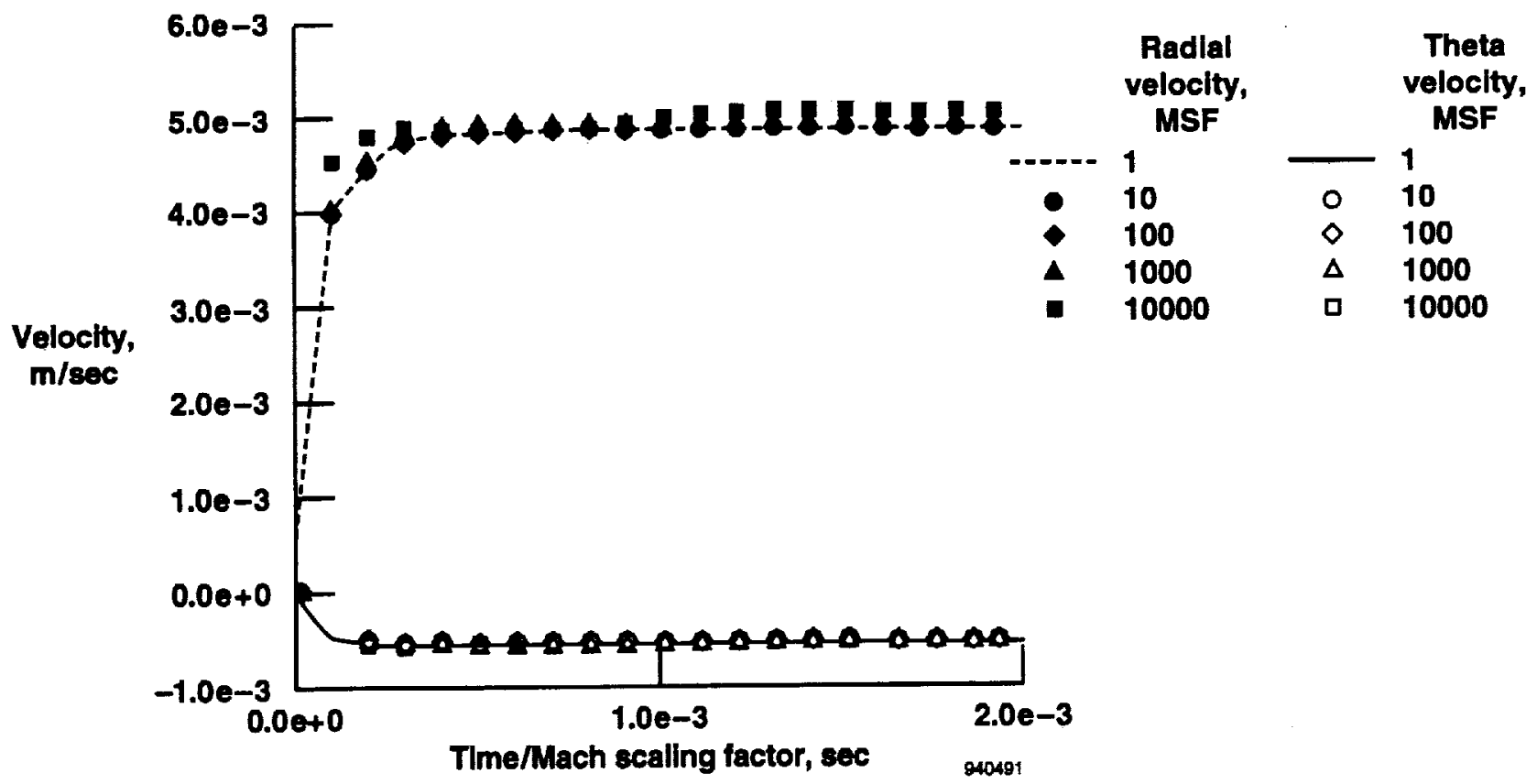

Figure 5. Numerical effect of scaling the Mach number in the liquid region. 


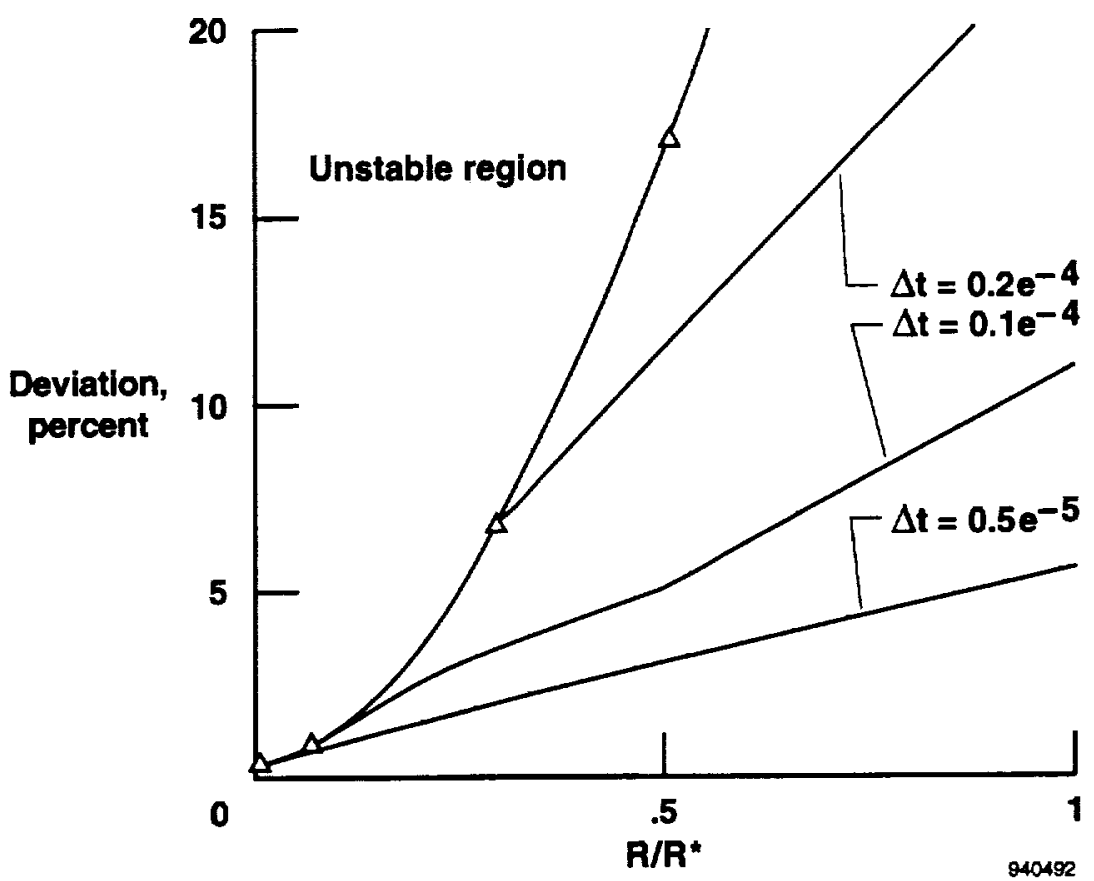

Figure 6. Numerical deviation of first-order correction to the pressure term in the gas region. (R/R* $=0$ implies no firstorder correction. $\mathbf{R} / \mathbf{R}^{*}=1$ implies an ideal first-order correction.)

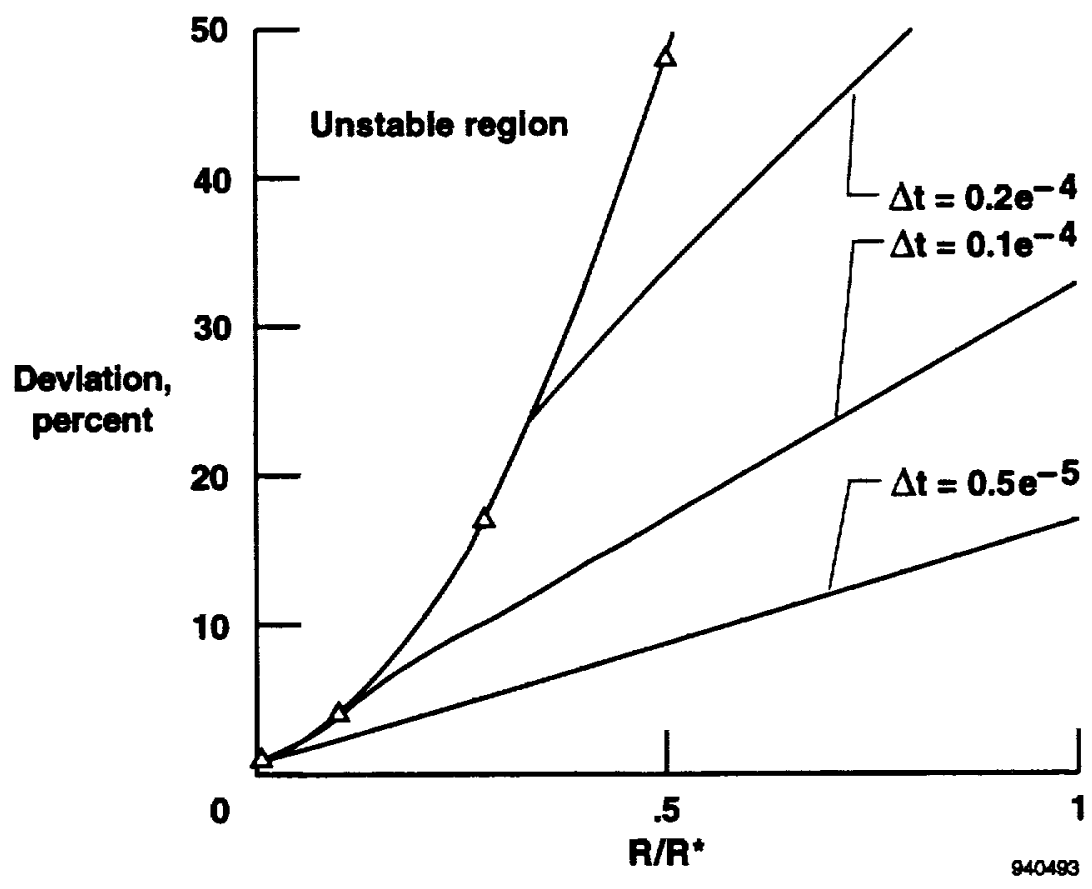

Figure 7. Numerical deviation of first-order correction to the pressure term in the liquid region. 


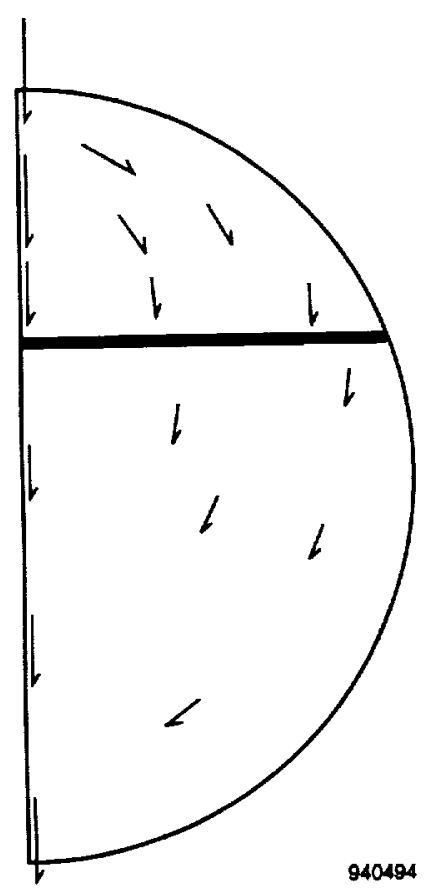

(a) Two-thirds.

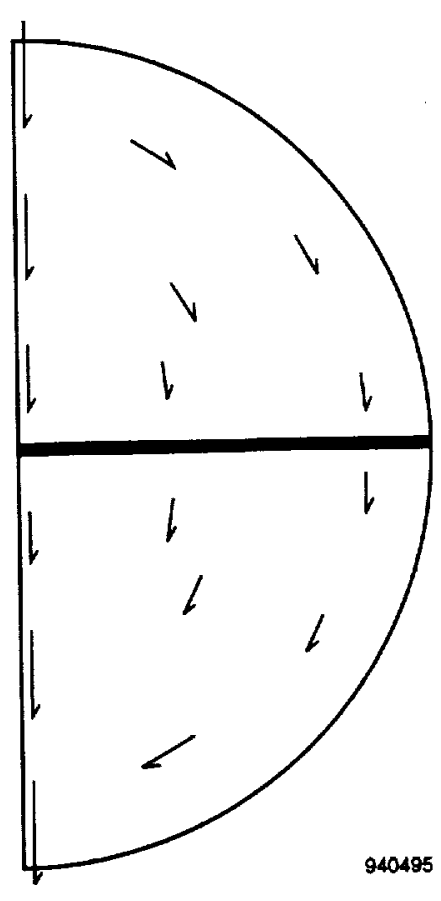

(b) One-half.

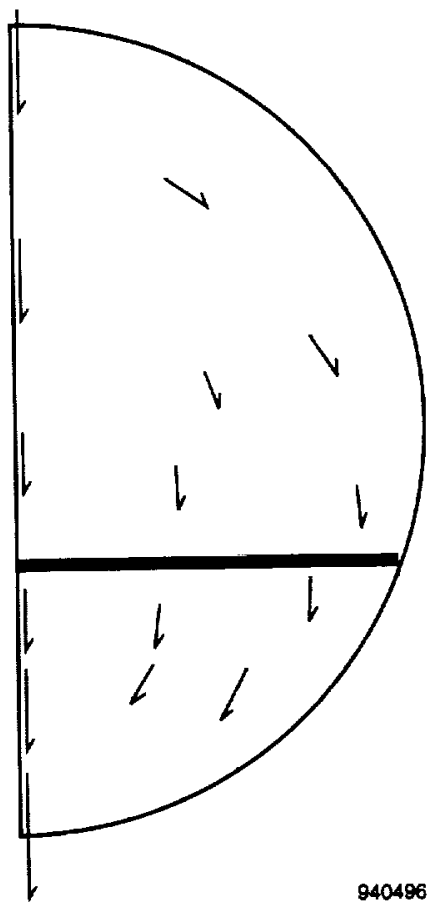

(c) One-Third.

Figure 8 . Velocity vectors at various liquid levels. 

Public reportingburdenforthiscolection of intormationisestimated toaverage thourperresponse. Includingthetimeforreviewinginstructions, searching exlstingdata sources, Publicrion collectionofiniomation, includingsuggestionsforreducingthisburden, to Washington Headquarters Services, Directoratefornformation OperationsandReports, 1215 Jeflerson Davis Highway Sulte 1204, Atington, VA 22202-4302, and to the Office of Management and Budgat, Paperwork Feduction Projoct (OTDA \begin{tabular}{|l|l|l}
\hline 1. AGENCY USE ONLY (Leave blank) & $\begin{array}{l}\text { 2. REPORT DATE } \\
\text { October } 1994\end{array}$ & $\begin{array}{l}\text { 3. REPORT TYPE AND DATES COVERED } \\
\text { Technical Memorandum }\end{array}$
\end{tabular}

4. TIRE AND SUBTILE 5. FUNDING NUMBERS

Numerical Modeling of a Cryogenic Fluid Within a Fuel Tank

\section{AUTHOR(S)}

Donald S. Greer

7. PERFORMING ORGANIZATION NAME(S) AND ADDRESS(ES)

NASA Dryden Flight Research Center

P.O. Box 273

WU 505-70-63

Edwards, CA 93523-0273

9. SPONSORINGMONOTORING AGEENCY NAME(S) AND ADDRESS(ES)

10. SPONSORINGMONTORING AGENCY REPORT NUMBER

National Aeronautics and Space Administration

Washington, DC 20546-0001

NASA TM-4651

11. SUPPLEMENTARY NOTES

Presented at the Second Thermal Structures Conference, Charlottesville, Virginia, October 18-21, 1994

12a. DISTRIBUTIONAVAILABILTYY STATEMENT

12b. DISTRIBUTION CODE

Unclassified-Unlimited

Subject Category 34

13. ABSTRACT (Maximum 200 words)

The computational method developed to study the cryogenic fluid characteristics inside a fuel tank in a hypersonic aircraft is presented. The model simulates a rapid draining of the tank by modeling the ullage vapor and the cryogenic liquid with a moving interface. A mathematical transformation was developed and applied to the Navier-Stokes equations to account for the moving interface. The formulation of the numerical method is a transient hybrid explicit-implicit technique where the pressure term in the momentum equations is approximated to first order in time by combining the continuity equation with an ideal equation of state.

\section{SUBJECT TERMS}

Compressible flow; Computation fluid dynamics (CFD); Fluid flow; Fluid Mechanics, Gas dynamics; Numerical modeling; Unsteady flow

\begin{tabular}{|l|l|l|}
\hline $\begin{array}{l}\text { 17. SECUATY CLASSIFICATION } \\
\text { OF REPORT } \\
\text { Unclassified }\end{array}$ & $\begin{array}{l}\text { 18. SECURTY CLASSIFICATION } \\
\text { OF THIS PAGE } \\
\text { Unclassified }\end{array}$ & $\begin{array}{l}\text { 19. SECUATY CLASSIFICATION } \\
\text { OF ABSTRACT } \\
\text { Unclassified }\end{array}$ \\
\hline
\end{tabular}

15. NUMBER OF PAGES

15

16. PAICE CODE

A03

20. LIMTAATION OF ABSTRACT

Unlimited 